\title{
Annual Outpatient Hysteroscopy and Endometrial Sampling (OHES) in HNPCC/ Lynch Syndrome (LS)
}

\section{Introduction and background}

Lynch Syndrome (LS) or HNPCC is a heritable syndrome caused by a mutation in one of the DNA mismatch repair (MMR) genes: MLH1, MSH2, MSH6, and PMS2.[1] Strict family history based diagnostic criteria (Amsterdam Criteria (AC)-II)[2] are used to identify people at increased risk. LS women have a $40-60 \%$ life time risk for endometrial cancer (EC). Some data suggest EC risk may be higher than that of colorectal cancer (CRC)[3,4] and greater for MSH2 and MSH6 than for MLH1 carriers.[5,4,6,3]

Gynaecological surveillance with endometrial sampling has been recommended from 30-35 years age in women who wish to delay/avoid risk-reducing surgery.[5,7] Unlike screening for CRC, the efficacy of EC screening remains unproven with only a handful of published series evaluating different modalities for EC screening in LS. These include: (a) Transvaginal (TVS) and/or transabdominal ultrasound;[8-10]; (b) TVS and endometrial sampling;[11,9] and (c) Hysteroscopy.[12,13]

The possibility of using hysteroscopy as a screening modality in LS was initially suggested in 2002.[14] However, no screen detected cancers have been reported in the published literature In this paper we report on our initial experience of annual outpatient hysteroscopy and endometrial sampling (OHES) as a screening strategy for EC in women with LS and compare it with TVS alone. 


\section{Methods}

The familial gynaecological cancer clinic at University College London Hospital is a tertiary clinic for managing women at 'high-risk' of gynaecological cancers. All women attending the clinic are managed by a multidisciplinary team, which includes gynaecological oncologists, clinical geneticist, clinical psychologist, radiologist, clinical nurse specialist, minimal access gynaecologist and pathologist. All women attending the clinic undergo detailed pedigree based risk-assessment and counselling. They receive comprehensive advice on advantages and disadvantages of risk-reducing surgery and screening, as well as reproductive and life style issues.

High-risk women with LS are identified on the basis of the diagnostic AC-II criteria[2] or the presence of a MMR gene mutation. LS women $>35-40$ years, who have completed their family, are offered risk-reducing surgery: total laparoscopic hysterectomy (TLH), bilateral salpingo-oophorectomy (BSO) and peritoneal washings. Women >35 years are offered screening for ovarian cancer within the United Kingdom Familial Ovarian Cancer Screening Study (UKFOCSS).

Since October-2007 eligible LS women attending the clinic are offered EC screening with TVS and OHES. Inclusion criteria are: LS women $>30$ years age. Exclusion criteria are: current pregnancy, prior hysterectomy, women opting for risk-reducing surgery or those negative on predictive testing for a known familial mutation. Women keep a menstrual calendar and undergo annual TVS with endometrial thickness (ET) measurement and OHES. TVS is performed by experienced ultrasonographers with over 10 years scanning experience. Hysteroscopy is carried out by one of a team of 3 experienced hysteroscopists, in an office setting, as described previously.[15] 
Endometrial sampling is undertaken in all women using guided biopsies/ polypectomy where indicated and/or a Pipelle device (Laboratoire C.C.D, France) at the end of the procedure.[15] Any irregular, heavy or unscheduled vaginal bleeding reported is investigated by bringing forward the annual OHES. Histological specimens are processed in total by a dedicated senior pathologist (EB). All data are stored on a bespoke database.

The current analysis includes all OHES procedures between October-2007 and March-2010. The primary outcome was EC and atypical endometrial hyperplasia (AEH). Inclusion of AEH as an outcome was based on it being an established premalignant lesion, lack of concordance in the diagnosis of AEH or EC between pathologists,[16] and 42.6\% of AEH having concurrent EC.[17] Wherever necessary, case notes, histopathology and TVS/biochemistry reports were reviewed. A screen positive on TVS is defined as (a) ET $>5 \mathrm{~mm}$ (measuring both endometrial layers): postmenopausal women,[18] (b) ET >12mm (measuring both endometrial layers in the second week of the cycle): premenopausal women,[10] (c) presence/suspicion of a polyp, or (d) irregular endometrium with fluid in the cavity. Although a cut off of 4 $\mathrm{mm}$ for endometrial thickness has been used by some to triage symptomatic postmenopausal women for hysteroscopy, we use a cut off of $5 \mathrm{~mm}$ in our practice, which is consistent with that advocated by a number of other institutions, a metaanalysis[18] and systematic review.[19] In addition, our experience of modelling endometrial thickness in asymptomatic post-menopausal women indicates the best performance characteristic may be obtained at a cut off of 5.1mm.[20] Cut-offs for endometrial thickness in asymptomatic premenopausal women unlike those for postmenopausal women are not well-defined. The $12 \mathrm{~mm}$ cut off was chosen on the 
basis of an earlier published report in the literature which evaluated TVS for screening in these women.[10] A screen positive at OHES is defined by the histology report.

Statistical analysis was undertaken using SPSS 12.0.1. The Mann-Whitney test was used to compare age distributions between groups after reviewing histograms. Fisher's test was used to calculate the difference between proportions. Kaplan Meier curves and the Log Rank Test was used to evaluate any difference in time to diagnosis. Two sided p values are reported for all statistical tests. Confidence intervals for a single proportion were calculated using the statistics package ‘Measuring Usability’ (J Sauro) LLC, Denver, Colorado, USA. Sensitivity, specificity, negative predictive value (NPV), positive predictive value (PPV), negative likelihood ratio (NLR) and positive likelihood ratio (PLR) were used to compare the screening performance for TVS and OHES.

\section{Results}

Between March-2004 and March-2010, 69 women with LS (fulfilling AC-II )[2] attended the clinic, of whom 25 were known mutation carriers (12MLH1, 12MSH2, 1PMS2). Thirteen women preferred risk-reducing surgery, five moved away, two were $<30$ years age and two tested negative for a familial mutation. The remaining 47 women opted for EC screening. Four eventually declined OHES and two have an appointment scheduled (Figure-1). The characteristics of the remaining cohort (41) are described in Table-1. 
Between October-2007 and March 2010, 41 prevalent and 28 incident screens (49.2 women screen years) were performed. The screening outcomes of the cohort are described in Figure-1. There was one failed outpatient hysteroscopy procedure which was completed under a general anaesthetic.

At prevalence screen, 39 were asymptomatic and 2 complained of irregular periods with intermenstrual bleeding. One of the symptomatic women (benign polyp) and 6 of the 39 (15.4\%) asymptomatic women had an abnormal TVS (2 endometrial polyps, 3 ET $>12 \mathrm{~mm}, 1$ irregular endometrium/fluid in the cavity) of whom 1 (polyp) had EC at histology. Three asymptomatic women were detected to have EC/AEH on OHES. Two of these three women with EC/AEH, underwent hysterectomy because of prevalence screen detected EC/AEH and 6 women opted for risk-reducing surgery after their initial prevalent screen. TVS and OHES in these 6 women were normal.

22 eligible women underwent incident screen-1 (Figure-1). Of these, 3 (13.6\%) presented with abnormal bleeding at 9-11 months from last screen. One had an in-situ levonorgestrel intrauterine system (LNG-IUS, Mirena ${ }^{\circledR}$ Schering Health Care). None had EC/AEH. All three had a normal TVS. Of the 19(86.4\%) asymptomatic women, TVS was abnormal in 5(26.3\%) which included the one woman with EC. This woman underwent hysterectomy following incident screen detected EC. Six of the 22 incident screen-1 women, underwent a subsequent second incident screen (incident screen-2) during the study time-period (Figure-1). Of these five were asymptomatic with normal TVS. However, endometrial hyperplasia (EH) was found at OHES in two of these cases. 
Overall 3 women (2 prevalence, 1 incident screen) had EC and one woman had AEH (prevalence screen) (Table-2). The latter includes one woman initially diagnosed as AEH who had a stage1a, grade1 EC at TLH BSO. Three of four women with screen detected endometrial pathology were MMR mutation carriers. No adjuvant treatment was needed for any of the three EC cases who remain recurrence free at 10, 16 and 22 months follow-up respectively. The woman with AEH opted for conservative management with a LNG-IUS (Mirena ${ }^{\circledR}$, Schering Health Care, UK). Follow-up OHES 4 months later was normal with no histological evidence of atypia/EC. No interval cancers have occurred in the cohort to date.

Combining incident and prevalent screens, for EC/AEH, OHES had similar specificity 89.8\%(95\%CI 79.2\%, 96.2\%) but higher PLR 9.8(95\%CI 4.6,21) and lower NLR (0 ) compared to TVS: specificity 84.75\%(95\%CI 73\%, 92.8\%), PLR 3.28(95\%CI 1.04,10.35) and NLR 0.59(95\%CI 0.22,1.58) (Table-3).

The median age of women with EC/AEH was 40.9 years (IQR 5.3 years) and the median age for the rest of the screened cohort was 43 years (IQR 12.3 years). EC/AEH cases were not found to differ from the rest of the cohort with respect to mutation status $(\mathrm{p}=0.64)$, number of screens $(\mathrm{p}=0.70)$ or age (Mann Whitney, $\mathrm{p}=0.40)$. However, mutation carriers undergoing screening had a significantly younger median age (39.7years, IQR 6.3) than the remaining AC-II positive women (44.2years, IQR12.8) $(\mathrm{p}=0.022)$. Kaplan Meier and Log Rank analysis of time to diagnosis for EC/AEH did not show any difference between known MMR carriers and those fulfilling AC-II alone ( $\mathrm{p}=0.85)$. 
Additional pathology found in this cohort included 6 endometrial polyps (3 prevalent and 3 incident). The annual incidence rate was 3.57\% (95\%CI 0.1, 18.35) for EC/AEH, 10.71\% (95\%CI 2.27, 28.23) for polyps, 7.1\% (95\%CI 0.88, 23.5) for EH (Table-4).

\section{Discussion}

This is to our knowledge the first series reporting the diagnosis of early stage EC in asymptomatic LS women using hysteroscopy based screening. Our findings indicate that OHES has high specificity and PLR and low NLR. The higher annual incidence rate of EC in our cohort $(3.57 \%$; 95\%CI 0.09,18.35) compared to the expected rate of approximately $1 \%$ in this population suggests that there may be lead time. OHES seems superior to TVS alone as a test for detecting EC/AEH in these women (Table3), with all four women with EC/AEH being detected by OHES compared to TVS which only detected two. The data further support the current guidelines that recommend that endometrial sampling should be undertaken in all LS women.[21,7]

TVS alone had a PLR of 3.28 for EC/AEH and NLR of 0.59 for EC/AEH. In contrast, OHES achieved an overall PLR of 9.8 for EC/AEH and a NLR of 0. Likelihood ratio combines information about sensitivity and specificity to assess test performance. It is independent of prevalence and permits comparisons across different types of tests. A value close to 1 has little clinical significance as the post-test probability (odds) of disease is little different from the pre-test probability. A PLR of $>5$ indicates moderate and $>10$ strong probability for disease being present when a test is positive. 
A PLR of $>5$ is generally a pre-requisite for adoption of a clinical test or procedure A

NLR of $<0.2$ is moderately and $<0.1$ strongly indicative of the disease being absent.

The results suggest that TVS alone as a screening test has poor ability to detect or rule out disease and is not very helpful in clinical decision making, while OHES has good diagnostic ability to detect disease and excellent ability to rule out disease in LS, making it an effective test for endometrial screening. For comparison the PLR and NLR for mammography are 9.4 and 0.19 [22], and for CT pulmonary angiogram in diagnosing pulmonary embolism are 8.6 and 0.06 respectively.[23] OHES performance in asymptomatic women in our series is consistent with the previously reported performance in symptomatic women (PLR 60.9, NLR 0.15) for diagnosing EC,[24] with a negative hysteroscopy reducing EC probability to 0.6\%.[24]

TVS alone would have missed 2 of 4 cases of EC/AEH, and did not seem to add to the performance of OHES. Our findings are consistent with a previous series using TVS and endometrial sampling which found that 55\%(6/11) of the screen detected EC, 50\%(2/4) AEH and 50\% of complex hyperplasia (4/8) cases had a normal TVS.[11] A majority of these false negative cases were post-menopausal. An increase in screen detected EC/AEH cases has been reported when TVS guided screening was replaced by a combined approach of TVS and endometrial sampling.[9] Overall our findings and these data suggest that in contrast to symptomatic postmenopausal general population women, the performance of TVS for detecting endometrial pathology in asymptomatic (pre and postmenopausal) LS women undergoing screening is poor. Reliable cut-offs for pre-menopausal and asymptomatic postmenopausal LS women are unknown. Two previous series have compared TVS alone 
to a combination of TVS and endometrial sampling and found the latter to be better at detecting EC/AEH.[9,11]

There are limitations to use of Pipelle endometrial sampling alone, including variability in its performance with menopausal status and type of pathology.[25] Reliable performance characteristics in asymptomatic LS women (significant proportion of who are premenopausal) are not available. Cancers have been missed on Pipelle alone, with three of the 11 cases of EC not detected on endometrial sampling in a previous LS series.[11]

The median age of our cohort was 42.9 (95\%CI 39.4, 49.7) years. Known MMR carriers undergoing screening were significantly younger than those fulfilling AC-II alone. This may reflect increased screening awareness among gene carriers and increased uptake of preventative surgery by older mutation carriers. The median age (40.9 years, IQR38.3, 43.5) of women diagnosed with EC/AEH in our study is significantly lower than that reported in two previous series using TVS and endometrial sampling: (a) 51.5 years (IQR 47,54, p=0.016),[11] median age of the entire cohort not reported and (b) 51 years (IQR 46,55, p=0.008 Mann-Whitney) with median age of cohort 46 years.[9] Our median age at diagnosis is also lower than that reported in epidemiological data (48-50 years), $[3,26]$ though it lies within the expected range (27-72 years) found in LS.[26,3] The small size of our data set makes it difficult to draw conclusions. If confirmed, age differences could be related to a combination of age and risk factor profile of our cohort and the lead time of screening. 
The strengths of our study include a strict screening protocol with prospectively collected data, longitudinal follow-up and description of outcomes for both prevalence and incident screens. The main weakness is the small size of the cohort and the need for validation in larger multicentre studies. It is to be noted that previous reports on LS screening using hysteroscopy have involved up to 62 patients and no EC were detected. Although we have compared TVS and OHES, the standard practice in a number of institutions is screening using a combination of TVS and endometrial sampling. TVS alone has a poor detection rate for endometrial pathology. A limitation of our study is a lack of ability to directly compare OHES with TVS and endometrial sampling. Future research in this area should be directed towards addressing this issue. Given the small sample size in most centres, this will necessitate a multicentre international study.

We are not aware of any reports on incidence rate of endometrial pathology in LS women. We found that 1 in 5 (incidence rate 21.4\%; 95\% CI 8.3, 40.1) LS women had endometrial pathology with an annual incidence of polyps of $11 \%$. This included one women with adenocarcinoma in a polyp. Our findings are in keeping with randomised trials in the general population which have shown that TVS and Pipelle have lower sensitivity for focal lesions like polyps compared to hysteroscopy.[27,28] Hysteroscopy detects lesions which may be missed by both TVS and Pipelle[28,27,15] and also permits directed biopsy from a focal lesion.

In the general population, up to 5\% of non-atypical $\mathrm{EH}$ and $30 \%$ of $\mathrm{AEH}$ progress to EC.[29] It is unknown if the rates of progression are greater in the LS population with a much higher a priori risk for EC. We can speculate that subsequent EC may have 
been prevented or delayed in some of the cases of polyps/hyperplasia treated in our series. EC/AEH was found in 9.76\% (95\%CI 2.7, 23.1) cases which is consistent with reports in the literature,[11,10] though higher than rates reported in some series.[8,12,13]

Both Hysteroscopy and Pipelle endometrial sampling are effective, easy to perform and well tolerated as outpatient procedures. Our failure rate of $1.45 \%(95 \% \mathrm{CI}$ 0.04,7.81) is consistent with those in systematic reviews $(4.2 \%, 95 \%$ CI 3.9,4.5).[24] The compliance with annual screening suggests that this is acceptable. A recent study evaluating acceptability of screening, found no significant difference in pain scores between either hysteroscopy or endometrial sampling.[30] The efficacy and patient acceptability of OHES is similar to the in-patient procedure and the former is more cost-effective.[31,32] Preliminary data suggest that hysteroscopy-based endometrial screening in LS does not appear to be associated with any psychological morbidity.[33]

\section{Conclusion}

LS women have a high incidence of polyps, premalignant lesions and EC. Initial data suggest that an OHES based surveillance strategy has good performance characteristics for detecting early stage EC/AEH in LS and may be superior to that of TVS alone. However, definitive data would require a larger international study. The challenges are the relatively small numbers of LS women at individual centres, the rates of risk reducing surgery and differences in use of routine outpatient hysteroscopy between countries. 
$-12$ 


\section{Acknowledgements}

We are particularly grateful to all our patients. We acknowledge the support and help provided by Ms S.Chamberlain (secretary), Ms S.Parker and Ms E.Palmer (nurse hysteroscopists) as well as The Eve Appeal. A large portion of this work was done at UCLH/UCL within the "Women's Health Theme" of the National Institute for Health Research UCLH/UCL CBRC supported by the Department of Health. 


\section{Disclosure of interests}

IJ has consultancy arrangements with Becton Dickinson, who have an interest in tumour markers and ovarian cancer. IJ and UM have a financial interest in Abcodia, Ltd., a company formed to develop academic and commercial development of biomarkers for screening and risk prediction. IJ is a member of the board of Abcodia Ltd and Women's Health Specialists Ltd. ANR has received honoraria from Fujirebio Diagnostics for giving lectures and attending meetings on the use of biomarkers in ovarian cancer management, but none were directly related to this work. ES received honoraria from Ethicon for provision of training to healthcare professionals; this was not related to this work. The other authors declare no conflict of interest.

\section{Contribution to authorship}

$\mathrm{RM}, \mathrm{AA}$ and $\mathrm{MJ}$ were involved in initial data collection. RM, UM were involved in analysis, drafting and writing of the paper. IJ, ANR, LS, ES, AA, SG, MJ, CB, EB contributed to writing of the manuscript. UM, IJ, ANR, RM, CB, LS, SG, ES, were responsible for the clinical care of the patients. EB reviewed the histological specimens and contributed to the histopathological sections of the manuscript. RM and UM performed the statistical analysis and contributed to writing the statistical sections of the manuscript. The final draft was prepared by RM, UM, IJ and approved by the others.

\section{Details of ethics approval}

The project was referred to the Chair of the Research Ethics committee (University College London Hospital, Research Deanery). Under the Research Governance 
Framework the project was deemed to be a clinical audit, and permission for data analysis and submission for publication was given on 26/04/2010.

\section{Funding}

This work has not been directly funded by any commercial organisation, charity, or other sources. A large portion of this work was done at UCLH/UCL within the “women's health theme” of the NIHR UCLH/UCL comprehensive biomedical research centre (CBRC) supported by the Department of Health.

\section{Copyright Statement}

This work is original and not under consideration for publication anywhere else. All the authors have contributed substantially to this paper and have approved the final version of the manuscript. Prof Usha Menon, the Corresponding Author of this article (the Contribution) has the right to grant on behalf of all authors and does grant on behalf of all authors, a license to the Archives of Gynecology and Obstetrics and its licensees, to permit this contribution (if accepted) to be published in the Archives of Gynecology and Obstetrics. 


\section{References}

1. Broaddus RR, Lynch PM, Lu KH, Luthra R, Michelson SJ (2004) Unusual tumors associated with the hereditary nonpolyposis colorectal cancer syndrome. Mod Pathol 17 (8):981-989

2. Vasen HF, Watson P, Mecklin JP, Lynch HT (1999) New clinical criteria for hereditary nonpolyposis colorectal cancer (HNPCC, Lynch syndrome) proposed by the International Collaborative group on HNPCC. Gastroenterology 116 (6):14531456

3. Aarnio M, Sankila R, Pukkala E, Salovaara R, Aaltonen LA, de la Chapelle A, Peltomaki P, Mecklin JP, Jarvinen HJ (1999) Cancer risk in mutation carriers of DNA-mismatch-repair genes. Int J Cancer 81 (2):214-218

4. Hendriks YM, Wagner A, Morreau H, Menko F, Stormorken A, Quehenberger F, Sandkuijl L, Moller P, Genuardi M, Van Houwelingen H, Tops C, Van Puijenbroek M, Verkuijlen P, Kenter G, Van Mil A, Meijers-Heijboer H, Tan GB, Breuning MH, Fodde R, Wijnen JT, Brocker-Vriends AH, Vasen H (2004) Cancer risk in hereditary nonpolyposis colorectal cancer due to MSH6 mutations: impact on counseling and surveillance. Gastroenterology 127 (1):17-25

5. Hendriks YM, de Jong AE, Morreau H, Tops CM, Vasen HF, Wijnen JT, Breuning MH, Brocker-Vriends AH (2006) Diagnostic approach and management of Lynch syndrome (hereditary nonpolyposis colorectal carcinoma): a guide for clinicians. CA Cancer J Clin 56 (4):213-225

6. Vasen HF, Stormorken A, Menko FH, Nagengast FM, Kleibeuker JH, Griffioen G, Taal BG, Moller P, Wijnen JT (2001) MSH2 mutation carriers are at higher risk of cancer than MLH1 mutation carriers: a study of hereditary nonpolyposis colorectal cancer families. J Clin Oncol 19 (20):4074-4080

7. Lindor NM, Petersen GM, Hadley DW, Kinney AY, Miesfeldt S, Lu KH, Lynch P, Burke W, Press N (2006) Recommendations for the care of individuals with an inherited predisposition to Lynch syndrome: a systematic review. Jama 296 (12):1507-1517

8. Dove-Edwin I, Boks D, Goff S, Kenter GG, Carpenter R, Vasen HF, Thomas HJ (2002) The outcome of endometrial carcinoma surveillance by ultrasound scan in women at risk of hereditary nonpolyposis colorectal carcinoma and familial colorectal carcinoma. Cancer 94 (6):1708-1712

9. Gerritzen LH, Hoogerbrugge N, Oei AL, Nagengast FM, van Ham MA, Massuger LF, de Hullu JA (2009) Improvement of endometrial biopsy over transvaginal ultrasound alone for endometrial surveillance in women with Lynch syndrome. Fam Cancer 8 (4):391-397

10. Rijcken FE, Mourits MJ, Kleibeuker JH, Hollema H, van der Zee AG (2003) Gynecologic screening in hereditary nonpolyposis colorectal cancer. Gynecol Oncol 91 (1):74-80

11. Renkonen-Sinisalo L, Butzow R, Leminen A, Lehtovirta P, Mecklin JP, Jarvinen $\mathrm{HJ}$ (2007) Surveillance for endometrial cancer in hereditary nonpolyposis colorectal cancer syndrome. Int J Cancer 120 (4):821-824

12. Lecuru F, Le Frere Belda MA, Bats AS, Tulpin L, Metzger U, Olschwang S, Laurent-Puig P (2008) Performance of office hysteroscopy and endometrial biopsy for detecting endometrial disease in women at risk of human non-polyposis colon cancer: a prospective study. Int J Gynecol Cancer 
13. Lecuru F, Metzger U, Scarabin C, Le Frere Belda MA, Olschwang S, Laurent Puig P (2007) Hysteroscopic findings in women at risk of HNPCC. Results of a prospective observational study. Fam Cancer 6 (3):295-299

14. Baxter NP, Duffy SR, Sheridan E (2002) Endometrial abnormalities in three sisters from a family with hereditary non-polyposis colorectal cancer syndrome. Bjog 109 (9):1076-1078

15. Gulumser C, Narvekar N, Pathak M, Palmer E, Parker S, Saridogan E (2010) Seeand-treat outpatient hysteroscopy: an analysis of 1109 examinations. Reprod Biomed Online 20 (3):423-429. doi:S1472-6483(09)00278-8 [pii]

10.1016/j.rbmo.2009.11.024

16. Zaino RJ, Kauderer J, Trimble CL, Silverberg SG, Curtin JP, Lim PC, Gallup DG (2006) Reproducibility of the diagnosis of atypical endometrial hyperplasia: a Gynecologic Oncology Group study. Cancer 106 (4):804-811

17. Trimble CL, Kauderer J, Zaino R, Silverberg S, Lim PC, Burke JJ, 2nd, Alberts D, Curtin J (2006) Concurrent endometrial carcinoma in women with a biopsy diagnosis of atypical endometrial hyperplasia: a Gynecologic Oncology Group study. Cancer 106 (4):812-819

18. Gupta JK, Chien PF, Voit D, Clark TJ, Khan KS (2002) Ultrasonographic endometrial thickness for diagnosing endometrial pathology in women with postmenopausal bleeding: a meta-analysis. Acta Obstet Gynecol Scand 81 (9):799816

19. Dimitraki M, Tsikouras P, Bouchlariotou S, Dafopoulos A, Liberis V, Maroulis G, Teichmann AT (2011) Clinical evaluation of women with PMB. Is it always necessary an endometrial biopsy to be performed? A review of the literature. Archives of gynecology and obstetrics 283 (2):261-266. doi:10.1007/s00404-010-1601-3

20. Jacobs I, Gentry-Maharaj A, Burnell M, Manchanda R, Singh N, Sharma A, Ryan A, Seif MW, Amso NN, Turner G, Brunell C, Fletcher G, Rangar R, Ford K, Godfrey K, Lopes A, Oram D, Herod J, Williamson K, Scott I, Jenkins H, Mould T, Woolas R, Murdoch J, Dobbs S, Leeson S, Cruickshank D, Skates SJ, Fallowfield L, Parmar M, Campbell S, Menon U (2011) Sensitivity of transvaginal ultrasound screening for endometrial cancer in postmenopausal women: a case-control study within the UKCTOCS cohort. The lancet oncology 12 (1):38-48. doi:10.1016/S14702045(10)70268-0

21. Vasen HF, Moslein G, Alonso A, Bernstein I, Bertario L, Blanco I, Burn J, Capella G, Engel C, Frayling I, Friedl W, Hes FJ, Hodgson S, Mecklin JP, Moller P, Nagengast F, Parc Y, Renkonen-Sinisalo L, Sampson JR, Stormorken A, Wijnen J (2007) Guidelines for the clinical management of Lynch syndrome (hereditary nonpolyposis cancer). J Med Genet 44 (6):353-362

22. Kerlikowske K, Smith-Bindman R, Ljung BM, Grady D (2003) Evaluation of abnormal mammography results and palpable breast abnormalities. Ann Intern Med 139 (4):274-284

23. Chu K, Brown AF (2005) Likelihood ratios increase diagnostic certainty in pulmonary embolism. Emerg Med Australas 17 (4):322-329

24. Clark TJ, Voit D, Gupta JK, Hyde C, Song F, Khan KS (2002) Accuracy of hysteroscopy in the diagnosis of endometrial cancer and hyperplasia: a systematic quantitative review. Jama 288 (13):1610-1621

25. Dijkhuizen FP, Mol BW, Brolmann HA, Heintz AP (2000) The accuracy of endometrial sampling in the diagnosis of patients with endometrial carcinoma and hyperplasia: a meta-analysis. Cancer 89 (8):1765-1772 
26. Vasen HF, Watson P, Mecklin JP, Jass JR, Green JS, Nomizu T, Muller H, Lynch HT (1994) The epidemiology of endometrial cancer in hereditary nonpolyposis colorectal cancer. Anticancer Res 14 (4B):1675-1678

27. Tahir MM, Bigrigg MA, Browning JJ, Brookes ST, Smith PA (1999) A randomised controlled trial comparing transvaginal ultrasound, outpatient hysteroscopy and endometrial biopsy with inpatient hysteroscopy and curettage. $\mathrm{Br} \mathrm{J}$ Obstet Gynaecol 106 (12):1259-1264

28. Pasqualotto EB, Margossian H, Price LL, Bradley LD (2000) Accuracy of preoperative diagnostic tools and outcome of hysteroscopic management of menstrual dysfunction. J Am Assoc Gynecol Laparosc 7 (2):201-209

29. Lacey JV, Jr., Sherman ME, Rush BB, Ronnett BM, Ioffe OB, Duggan MA, Glass AG, Richesson DA, Chatterjee N, Langholz B (2010) Absolute risk of endometrial carcinoma during 20-year follow-up among women with endometrial hyperplasia. J Clin Oncol 28 (5):788-792. doi:JCO.2009.24.1315 [pii]

10.1200/JCO.2009.24.1315

30. Elmasry K, Davies AJ, Evans DG, Seif MN, Reynolds K (2009) Strategies for endometrial screening in the Lynch syndrome population: a patient acceptability study. Fam Cancer 8 (4):431-439

31. Kremer C, Duffy S, Moroney M (2000) Patient satisfaction with outpatient hysteroscopy versus day case hysteroscopy: randomised controlled trial. Bmj 320 (7230):279-282

32. Saridogan E, Tilden D, Sykes D, Davis N, Subramanian D (2010) Cost-Analysis Comparison of Outpatient See-and-Treat Hysteroscopy Service with Other Hysteroscopy Service Models. J Minim Invasive Gynecol 17 (4):518-525. doi:S15534650(10)00121-4 [pii]

10.1016/j.jmig.2010.03.009

33. Wood NJ, Munot S, Sheridan E, Duffy SR (2008) Does a "one-stop" gynecology screening clinic for women in hereditary nonpolyposis colorectal cancer families have an impact on their psychological morbidity and perception of health? Int J Gynecol Cancer 18 (2):279-284 


\section{Word Count}

2583

\section{TABLES LIST}

Table 1: Characteristics and outcome of Lynch Syndrome (LS) women undergoing OHES screening for endometrial cancer

*One patient had a MLH1 VUS (variant of uncertain significance) AC-Amsterdam Criteria, AEH-atypical endometrial hyperplasia, OHES-Outpatient Hysteroscopy and Endometrial Sampling, IQR-Interquartile range

Table 2- Screening details in cases of screen detected abnormal endometrial pathology

* Benign endometrial polyps were diagnosed at OHES alone on 2 consecutive screens

** Diagnosis confirmed following total laparoscopic hysterectomy and bilateral salpingo-oophorectomy

AEH-atypical endometrial hyperplasia, BSO-Bilateral salpingo-oophprectomy; ECEndometrial cancer; EP-endometrial polyp; ECxP-endocervical polyp; Gr-Grade, OHES -Outpatient Hysteroscopy and Endometrial Sampling; P-prevalent screen; Iincident screen; MMR-mismatch repair gene, TLH-Total Laparoscopic Hysterectomy, VUS-variant of uncertain significance 


\section{Table-3: Performance Characteristics of OHES and TVS}

CI-confidence interval, FN-false negative, FP-false positive, I-incident, NLR-negative likelihood ratio, NPV-negative predictive value, OHES-Outpatient Hysteroscopy and Endometrial Sampling, P-prevalent, PLR-positive likelihood ratio, PPV-positive predictive value, TN-true negative, TP-true positive, TVS-transvaginal scan

Table 4- Abnormal pathology rates (per patient) at Prevalent and Incident screening in the cohort

\footnotetext{
* Endometrial polyps diagnosed on 2 consecutive screens in one case

** One case with endometrial cancer also had a malignant endometrial polyp

\# Includes 22 women, 6 of whom underwent a third round of annual screening
}

\section{Figure 1 legend: Consort flow chart for the cohort}

AEH-Atypical endometrial hyperplasia, BSO-Bilateral salpingo-oophprectomy, DNA-did not attend, EC-endometrial cancer, ECxP-endocervical polyp, EHendometrial hyperplasia, EP-endometrial polyp, LS- Lynch Syndrome, N-normal, OHES-Outpatient Hysteroscopy and Endometrial Sampling, Pos-positive, TLH-Total Laparoscopic Hysterectomy, TVS-transvaginal scan 